
Not for reproduction, distribution or commercial use.

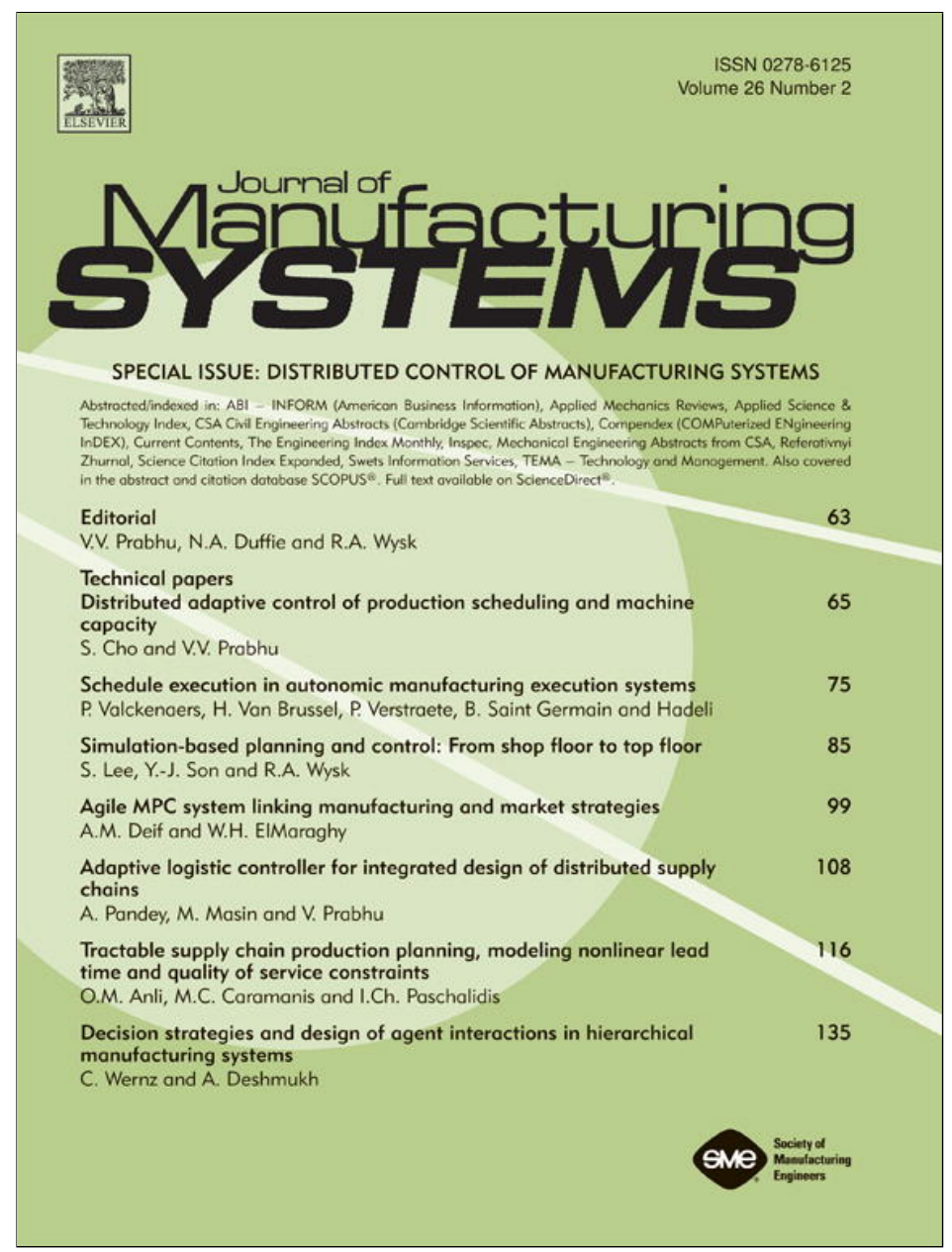

This article appeared in a journal published by Elsevier. The attached copy is furnished to the author for internal non-commercial research and education use, including for instruction at the authors institution and sharing with colleagues.

Other uses, including reproduction and distribution, or selling or licensing copies, or posting to personal, institutional or third party websites are prohibited.

In most cases authors are permitted to post their version of the article (e.g. in Word or Tex form) to their personal website or institutional repository. Authors requiring further information regarding Elsevier's archiving and manuscript policies are encouraged to visit:

http://www.elsevier.com/copyright 
Technical paper

\title{
Schedule execution in autonomic manufacturing execution systems
}

\author{
Paul Valckenaers*, Hendrik Van Brussel, Paul Verstraete, Bart Saint Germain, Hadeli \\ Mechanical Engineering Department, K.U. Leuven, Belgium
}

\section{A R T I C L E I N F O}

\section{Article history:}

Received 31 August 2006

Received in revised form

17 April 2007

Accepted 14 December 2007

\begin{abstract}
A B S T R A C T
This paper discusses a manufacturing execution system (MES) that prefers and attempts to follow a given schedule. The MES performs this task in an autonomic manner, filling in missing details, providing alternatives for unfeasible assignments, handling auxiliary tasks, and so on. The paper presents the research challenge, depicts the MES design, and gives experimental results. The research contribution resides in the novel architecture in which the MES cooperates with schedulers without inheriting the limitations of the world model employed by the scheduler. The research forms a first development, and a list of further research is given.
\end{abstract}

(C) 2008 The Society of Manufacturing Engineers. Published by Elsevier Ltd. All rights reserved.

\section{Introduction}

Decentralized manufacturing control systems have been successfully demonstrating robustness and reconfigurability [1-5]. However, regarding system-wide overall optimization there remains significant margin for improvement. A key issue is the (in)ability of such systems to plan ahead in time: decision myopia [6]. A first step in this regard is the ability to predict the nearfuture behavior of the system [7]. Alternatively, a market-based decentralized control system may use learning and adaptation to remedy its decision myopia within sufficiently stable manufacturing environments [8]. But even when the decentralized control system anticipates the consequences of its decisions (and may reconsider them), centralized scheduling technology retains superior abilities to optimize the overall system. In contrast, centralized scheduling has failed to provide robust, detailed, and complete solutions (see further).

The above situation has prompted the design of manufacturing execution systems (MES) that account for a given schedule but retain their robustness and flexibility. Actually, a team of skilled human operators and shop floor managers - using a given schedule as an initial guideline but handling all details and contingencies - is the most common MES in industrial practice. This already is an MES belonging to the class of systems envisaged by the research in this paper. The research in this paper discusses the first developments aimed at providing the same functionality in an autonomic holonic MES. In this context, autonomic implies that the MES keeps functioning

\footnotetext{
* Corresponding address: Mechanical Engineering Department, Room - 04.72, Afd. CIB, Celestijnenlaan 300A - Bus 2422, 3001, Heverlee, Belgium. Tel.: +32 16322 483.

E-mail address: Paul.Valckenaers@mech.kuleuven.be (P. Valckenaers).
}

regardless of the availability and quality of the given schedule (cf. www.researchweb.watson.ibm.com/autonomic/overview).

The paper first discusses the research challenge: cooperating scheduling and manufacturing execution systems. Next, the holonic MES is presented concisely. This control system has been adapted and extended to prefer executing a given schedule. Subsequently, the experiments - testing these adaptations against various schedules - and their results are presented and discussed. Finally, conclusions are given and future research is discussed.

Importantly, note that the contribution of this paper resides in the novelty of the overall architecture: a cooperating MES that provides MES functionality while accounting for an externally supplied schedule. This architecture allows an MES developer to add whatever MES functionality happens to be required. Examples of concerns that are often not addressed by a scheduler are exotic product variants, maintenance tasks, reverse logistics for product carriers, transportation and storage of products and tooling, sequence-dependent processing times in combination with external setups, nondeterministic process plans, and so on. In contrast to schedulers, MESs prefer sufficiently accurate world models over optimized decisions.

This distinguishes the research in this paper from rescheduling research in a fundamental manner. Indeed, rescheduling remains constrained by its modeling formalism. A scheduler will be ignorant of activities that its model, defining the problem, fails to capture. If the MES needs to adapt or complete a given schedule because the scheduler ignores some pertinent facts, rescheduling will not provide a full solution. At best, rescheduling compensates for the drift between reality and the perception thereof within the (re)scheduler.

Autonomic MES and rescheduling are not competing, but cooperating. Rescheduling makes the MES task easier because the schedule will be more realistic and feasible. The MES allows the scheduler to solve easier problems because it will fill in the missing 
pieces and make final adjustments. Moreover, a holonic MES (see further) predicts future system states, allowing the scheduler to start with an estimate of the system's state when its results become available; computationally more expensive scheduling techniques may be utilized thanks to this MES service.

\section{Problem statement}

The research discussed in this paper aims at combining scheduling and MES technology, accounting for their respective weaknesses and strengths. Therefore, the following paragraphs discuss the weak points of the technologies and state the ensuing challenge of combining both in a manner that negates these deficiencies. Note that the weaknesses, discussed below, are intrinsic and are extremely unlikely to be remedied through research and development within the reader's lifetime.

\subsection{Scheduling weaknesses}

Today, manufacturing management wants to combine the advantages of scheduling with the robustness and agility of decentralized manufacturing execution systems. Scheduling and planning systems are predominantly centralized systems that promise optimized performance. However, this optimization functionality has some significant caveats. Schedulers rely on an optimization technique (e.g., linear programming). This scheduling technique will be computationally efficient ( $a$ fortiori) at the cost of solving only an approximation of the real problem, which is NPhard. This is reflected by the technique requiring the scheduling problem to be formulated in a suitable format (e.g., specifying machine capacity per time bucket, process step sequences for products, and so on). Typically, this results in the following limitations:

- Some manufacturing activities will not be modeled. For instance, the schedule may ignore the need to return empty transportation containers to the locations where they are to be loaded.

- Some manufacturing activities are modeled very coarsely. For instance, transportation tasks may be assumed to have a fixed lead time. Likewise, the fluctuations of machine utilization within a time bucket may be invisible to the scheduler. Both approximations regularly cause the user to reserve excess capacity to account for the (almost) worst case.

- Some manufacturing activities are decided up front. For example, the shift schedule of the workforce may be given instead of optimized.

- Some product attributes are not modeled or only very coarsely. For instance, a scheduler may ignore product types while balancing product flows.

- The model may ignore constraints. For example, some large product variants may not be processed simultaneously in neighboring workstations (not enough space).

- The model may add constraints. Many scheduling techniques cannot handle alternatives in process plans.

- The models may not represent inherent uncertainties. In disassembly operations, for example, the outcome typically will be stochastic, resulting in refurbishable components or scrap material.

- Model refinement possibilities are restrained by limitations of the models' expressive power.

- The model may only be able to represent the actual user objective indirectly and approximately (e.g., throughput maximization approximated by makespan minimization).

These limitations have multiple causes. The model type simply may be unable to represent a more accurate reflection of re- ality (typical for the more efficient scheduling techniques). Alternatively, too much details may require too much computational efforts (e.g., typical for AI planners). Brute-force model refinements (e.g., reducing the time bucket size) often cause computational efforts to rise sharply. This imposes limitations on the possibilities to refine and expand models.

Moreover, schedulers use limited models because of the required modeling efforts and the required modeling expertise. $A$ key issue in this respect is model maintenance. These modeling efforts by skilled people would be a surmountable challenge if the resulting models would be long-lived and reusable. Unfortunately, scheduling models typically exhibit poor transferability in space (from one factory to another) and time (to the same factory in the future). The bad news is that there are mechanisms that actively cause this undesirable situation, as follows:

- The scheduling model needs to accurately reflect the performance-determining aspects of the production system (bottlenecks). The remainder is modeled as good as possible.

- The performance-limiting elements in a production system are the prime targets for improvements.

- Because scheduling is efficiently solving an approximation of an NP-hard problem, the model needs frequent adaptation to changed situations. The approximations are inherently not robust.

In other words, a professional plant manager is constantly rendering the scheduling model obsolete while allocating investments properly. In addition, a dynamic manufacturing environment regularly shifts the operating point around which this model should be constructed. In practice, applying scheduling technology becomes increasingly more difficult when the distance between reality and the scheduling model shrinks. Schedulers can draw the overall course of action for production activities at aggregated levels, but they can become a maintenance ordeal when put in charge of detailed manufacturing execution management. Detailed scheduling is viable as long as the financial returns cover the model maintenance costs, which explains why detailed scheduling is largely confined today to high-investment industries (oil, steel, silicon).

\subsection{Decentralized MES weaknesses}

A manufacturing execution system - MES - sacrifices optimality to ensure traceability and expressive power (for the embedded models). In practice, MES model accuracy and completeness is far from perfect, but it is a priority. An MES will reflect reality to a sufficient degree, allowing operational management to track, trace, and command all production activities. As technology progresses and the cost of sensors and communication decreases, the MES models become more refined, accurate, and complete. Existing MES and manufacturing control systems often balance decisionmaking mechanisms against model expressiveness. This paper highlights how such compromises may no longer be necessary. The research results discussed in this paper emphasize the embedded models' expressive power - supporting a one-to-one correspondence to real-world entities and activities (maps) because it offers significant system integration, reusability, and model maintenance advantages. This issue is, however, not further addressed in this paper [9].

The downside of the above is that it fails to optimally solve the production management problem, which is NP-hard. In particular, a decentralized MES will neglect approximating the global optimum when its embedded local and myopic optimizations fail to address the overall performance. Proper tuning of the local decision mechanisms may provide this, but it shares the transferability (in time and space) difficulties and maintenance concerns with the centralized scheduling alternatives. And, 
decentralization renders this more difficult. The key issue is to coordinate the local mechanisms efficiently and effectively without losing the advantages of decentralized manufacturing control (swift and meticulous handling of local situations).

\subsection{Decentralized MES challenges}

Ideally, a decentralized MES will utilize an optimized schedule as an initial guideline from which the detailed local course of action is derived. Requiring the decentralized mechanisms to align their decisions from scratch is inherently inefficient in the presence of a suitable schedule, even if this schedule is aggregated and incomplete. This initial schedule may be generated by a scheduler or derived from the past production records.

In any case, the MES has to complete, correct, and adapt the schedule to make it feasible, complete, and adapted to the actual situation. Indeed, the discussion of scheduler weaknesses above comprises a list of elements that the MES needs to handle. Typically, the initial schedule only specifies the main activities at an aggregated level. The MES needs to elaborate those in more detail and add the missing activities (transport, storage, material supply, and so on). Moreover, the MES cannot rely blindly on the given schedule but needs to critically assess which information is valid and valuable, which information is stale, incorrect, and so forth. In other words, the MES needs to execute it in an autonomic fashion. This paper discusses initial developments in this direction.

\subsection{Related work}

As stated in the introduction, the contribution of this paper resides in the overall architecture. Work by others on rescheduling is not relevant in this perspective, short of developments that address the problem modeling task. Today, this task requires significant efforts - often many persons-months - by highly skilled human experts. In this respect, the autonomic capabilities of the MES must enable it to cooperate even with poorly maintained scheduling systems and scheduling inputs based on recordings of 'similar' production runs in the past, reflecting industrial practice and needs. This decoupling is an important achievement of the research in this paper.

Work on decentralized manufacturing control suffers from decision myopia [6]. Lin and Solberg [3] used a market mechanism to allocate the available production capacity more optimally. AARIA [4] is a comprehensive framework to use negotiation protocols and pricing for allocating production capacity along a production chain. Bussmann [2] also uses negotiation protocols to allocate workpieces to workstations but uses queue length, buffer space, and process availability instead of price. The latter system is implemented in an existing factory within the automotive industry. Typically, all these systems use a variation on the wellknown contract net protocol [10]. All of these approaches are myopic; they share this property with market mechanisms. Efforts to remedy the myopia produced valuable results but fail to match the optimization contributed by scheduling technologies $[7,8]$.

Work on schedule execution, as targeted in this paper, is rare and typically has some hierarchical architecture. Bongaerts [11] combines hierarchical scheduling with local autonomy that is assisted by information about rescheduling margins and sensitivities. The ADACOR architecture supports switching between a heterarchical configuration, to handle disturbances, and a hierarchical configuration, optimizing normal operations [12]. Unfortunately, such designs easily inherit the drawbacks of scheduling systems concerning the modeling of reality, which prompted the design in this paper to refrain from hierarchical relationships with scheduling systems. Bongaerts [11] and Leitao [12] mainly contribute to the agility of the control system in face of disturbances. Control system maintenance remains an unsolved issue.

Work on emergent synthesis (e.g., [13]) was very relevant for the design of the starting point of the research in this paper. The contribution in this paper consists of making the emergent behavior more prescribable and, it is hoped, more predictable by reducing the functionality that needs to emerge. In contrast to the research in this paper, work by others on emergent synthesis aims to avoid the need for explicit planning, scheduling, or programming of control systems. This focus is probably motivated by past experience in which combinations of scheduling and execution systems have inherited the maintenance concerns of a scheduling system.

Finally, concerning agent architectures, it is important to notice that there are two different research targets. PROSA (see further) and ADACOR are system architectures that address the composition, structure, and dynamics of a society of agents. The BDI framework is the best-known example of an agent architecture (cf. www.agentlink.org/roadmap/al3rm.pdf) addressing the internal structure and operations of an individual agent. As an analogy, BDI defines the internal structure of a single room whereas PROSA or ADACOR would define the elements of a university campus, a holiday resort, and so on. System architectures, such as PROSA and ADACOR, refrain from imposing an internal architecture for individual agents.

Some researchers believe that the BDI framework alone is insufficient to handle all of the needs of agent societies corresponding to full applications $[14,15]$. In the hotel analogy, this would become apparent during the awkward job of fitting the hotel restaurant and kitchen into a collection of standard guest rooms. A specific issue is that BDI agents keep their world model (beliefs) to themselves, forfeiting the benefits of stigmergic designs, but this discussion is out of scope for this paper [16].

\section{Holonic MES}

The MES research discussed in this paper strongly emphasizes problem domain modeling as part of building a manufacturing control solution; this concern takes precedence over devising highperforming decision-making mechanisms. The expressive power of the models embedded in the holonic MES supports a one-to-one correspondence with the entities and activities in the production system [17].

Because any computationally efficient decision-making mechanism - solving an inherently NP-hard problem - will have a smaller applicability range than the constituents of the problem domain model, the holonic MES postpones the introduction of these mechanisms until the final stages of the control system formation. Actually, the decision-making mechanisms are considered easily replaced plug-in components that are added last and can be updated regularly without causing maintenance or inducing modification requirements to the remainder of the control system. This design is highly suited for answering the above MES challenges.

This section first presents the holonic MES design (Sections 3.1 and 3.2). Next, it presents how the decision-making mechanisms will be biased toward executing a given schedule (Section 3.3). Section 4 presents and discusses experiments and results.

\subsection{PROSA}

The holonic MES implements the PROSA reference architecture [7]. This reference architecture acronym denotes ProductResource-Order-Staff Architecture. The structure of the systems designed along the PROSA architecture is composed of three types of basic agents: 
- Resource agents correspond to a physical part - e.g., a production resource in the manufacturing system - and contain an information processing part that controls their resource. They offer processing capacity and functionality to the surrounding agents. In manufacturing control systems, a resource agent is an abstraction of the production means, such as machines, conveyors, pallets, raw materials, tool holders, material storage, personnel, floor space, and so on. In objectoriented terminology, each resource agent reflects a physical resource, is able to drive this resource, and keeps its resourcestate-reflecting attributes synchronized with this resource's actual state.

- Order agents represent tasks in the underlying system. They are responsible for performing the assigned work correctly and on time. In manufacturing control systems, an order agent manages the physical product being produced, the productstate model, and all the logistic information processing related to the job. An order agent may represent customer orders, make-to-stock orders, prototype-making orders, orders to maintain and repair resources, and so on.

- Product agents hold the process and product knowledge to assure the correct making of a product with sufficient quality. As such, they contain the product model of their product type, not the product-state model of one physical product instance being produced. The product agent acts as an information server to the other agents, delivering the right recipes in the right place. It knows how to make a product without making any decisions about when and where production takes place. In an advanced application, a product agent may even redesign the product in response to requests from the order agent. Note that human experts are likely to be part of such an agent. This actually happens during production of large buildings, cruise ships, and so on, to solve problems discovered during construction.

These basic agents are structured through aggregation and specialization. An agent may belong to several aggregations, and aggregated agents can dynamically change their contents. For instance, membership of a batch order agent may depend on the timely arrival of the prospective members. Aggregated agents may emerge out of the self-organizing interaction of agents or they may be designed up front. The number of aggregation levels depends on the specific needs of a certain system and is not dictated by the architecture. Aggregation reduces exposure of the individual agents and increases software reuse. For instance, a shopping list agent simply creates new agents for each item on the shopping list and manages the aggregate; the production of the individual items is delegated to the corresponding item agents.

Specialization allows a layered implementation of the agents, where the higher layers can be shared and, more importantly, used as interfaces to the remainder of the coordination and control system. For instance, in a manufacturing control system there will be a specialization hierarchy starting from the agent along resource agent, transport resource agent, toward conveyor belt agent. Importantly, any agent working with resource agents will perform its duties regardless of which more specialized kinds of resource agents it is interacting with.

Staff agents, as in human organizations, can be added to assist the basic agents with expert knowledge. These agents often reflect functions such as planning systems. However, they only produce advice and never hold final responsibilities concerning command and control of the underlying system. In certain ways, these agents reflect information rather than being a function. The weak coupling (advice-only) effectively limits the mutual exposure of the agents and eases integration.

Furthermore, relentless usage of delegation characterizes the interaction amongst the basic PROSA agents, which limits their
Table 1

Sample resource agents (corresponding part in underlying system)

\begin{tabular}{ll}
\hline Manufacturing & Supply networks \\
\hline Material receiving station & Raw material supplier \\
Drilling workstation & Manufacturing plant \\
Thermal treatment workstation & Repackaging facility \\
Packaging station & Wholesale distributor \\
Shipment station & Shops/Stores \\
Automatic storage and retrieval system & Distribution center \\
FIFO pallet buffer & Truck fleet \\
Part buffer & Truck \\
Overhead crane & Aircraft \\
Conveyor belt & Container \\
AGV fleet & Pallet \\
\hline
\end{tabular}

Table 2

Sample product agents

\begin{tabular}{|c|c|c|}
\hline Domain & Product type & Recipe or product agent description \\
\hline Manufacturing & $\begin{array}{l}\text { Heat treatment of } \\
\text { gear wheels }\end{array}$ & $\begin{array}{l}\text { The product agent knows which } \\
\text { furnaces are suited and which } \\
\text { temperature trajectory is required for } \\
\text { the heat treatment in each of the } \\
\text { candidate furnaces. }\end{array}$ \\
\hline Manufacturing & $\begin{array}{l}\text { Heat treatment of } \\
\text { gear wheels } \\
\text { (advanced) }\end{array}$ & $\begin{array}{l}\text { In addition to the above, the product } \\
\text { agent knows the set of allowed } \\
\text { temperature trajectories, creating the } \\
\text { opportunity to batch product instances } \\
\text { of its type with product instances of } \\
\text { compatible types. }\end{array}$ \\
\hline $\begin{array}{l}\text { Supply } \\
\text { networks }\end{array}$ & $\begin{array}{l}\text { Heat treatment of } \\
\text { gear wheels }\end{array}$ & $\begin{array}{l}\text { The product agent knows which } \\
\text { manufacturing plants offer the } \\
\text { required processing facilities and } \\
\text { knows how to route its product } \\
\text { instance through the supply network } \\
\text { such that it visits a suitable plant and } \\
\text { receives its heat treatment. Inside the } \\
\text { plant, the above product agent takes } \\
\text { over. The product agent knows which } \\
\text { containers are needed for transporting } \\
\text { the product instance. }\end{array}$ \\
\hline
\end{tabular}

Table 3

Sample order agents

\begin{tabular}{|c|c|c|}
\hline Domain & Task & Remarks \\
\hline Manufacturing & Customer order no. 332 & $\begin{array}{l}\text { Tracks and manages a specific } \\
\text { customer order with the } \\
\text { assistance of the product agent } \\
\text { within the plant as a rush order. }\end{array}$ \\
\hline Supply network & Customer order no. 556 & $\begin{array}{l}\text { Tracks and manages a specific } \\
\text { customer order with the } \\
\text { assistance of the associated } \\
\text { product agent within a supply } \\
\text { network. }\end{array}$ \\
\hline
\end{tabular}

exposure to system properties outside their own scope. Product agents never attempt to manage state information about ongoing tasks and delegate this to the order agent. Product agents receive an object representing the state of a task from the corresponding order agent whenever they need this information to perform their tasks. Likewise, order agents always consult product agents about possible ways to proceed with their task.

Finally, three tables list, respectively, sample resource agents (Table 1 ), product agents (Table 2 ), and order agents (Table 3 ). The applicability range of PROSA is online real-time resource allocation and the coordination of the related activities.

\subsection{Ant agents}

The holonic MES uses a bio-inspired design to search and select solutions to carry out the required manufacturing orders. And, 
the MES generates short-term forecasts at the same time. These forecasts predict resource loads and order routings, including their timing, based on the current order intentions and accounting for the latest information available $[7,17]$. This section first discusses an analogy to clarify the underlying principles on more familiar grounds (for most readers). Next, it introduces the ant agents executing the coordination and forecasting in a manufacturing setting.

\subsubsection{Travel arrangement analogy}

To clarify how the MES handles production requirements and produces short-term forecasts in resource allocation, this paragraph utilizes an analogy: human agents making travel arrangements. Next, it discusses how an agent system may achieve similar results, compensating its limitations and benefiting from its strengths relative to the human agents. In the MES, the travelers are not humans but production orders. Note that each order agent arranges its own production as humans would arrange their journey individually.

When a human travel agent arranges a vacation on behalf of a customer, the agent first checks for availabilities with the service-offering resources: airlines, hotels, event organizers, and so forth. In the MES, the order agent checks availabilities with the service-offering resource agents in the factory: machining stations, conveyor belts, storage spaces, inspection stations, packaging stations, and so on. The human travel agent accounts for the customer requirements. The MES order agent checks with its product agent about the processing requirements that must be met. These inquiries typically investigate several options and possibilities. While making these inquiries, the agent receives answers accounting for a prediction of resource availabilities (that is, the current reservations roster of the resource).

This resource availability prediction is constructed based on the reservations by agents representing travelers. In other words, the resources constitute an environment in which order agents deposit their intentions and commitments, and the (reservation departments of the) resources combine such information to construct forecasts of their utilization in the future. This environment transcends the present in a manner that accounts for the overall system state, including mental states that have been externalized into the environment.

Our travel agent combines customer requirements/preferences with the answers from its inquiries to construct a 'best' travel arrangement, and makes reservations at the resources involved. In this manner, the forecasts are adapted to the new situation. In case of disturbances (e.g., a fire destroys the hotel), the agent is informed by the resource, which triggers actions to deal with the situation.

Software agents have limited reasoning capabilities compared to humans. In contrast, they have fewer problems with performing simple tasks at high frequencies. The multi-agent design based on the above behavior pattern is therefore different in the following ways:

- The agent system requires frequent reconfirmation of reservations. Humans consider it a burden to reconfirm their flight once. Software agents easily reconfirm their reservations every 10 seconds. This enables the overall system to see changes and disturbances very rapidly without requiring a reliable notification system. Reservations that are not reconfirmed for two minutes can safely be discarded, and the resource becomes again available. Likewise, the software agent detects any disturbance at a resource during reconfirmation.

- The software agents cannot distinguish critical aspects from less important ones. To have a reliable service with little chance of nasty surprises, the agents virtually execute complete travel trajectories in detail, checking and making arrangements with all resources involved in the journey. Instead of knowing which elements need prior arrangement (hotel, flight), the agent makes arrangements with all resources involved. Since these agents not only arrange travel beforehand but also remain in charge during execution, they need to manage this anyhow. Handling many simple tasks is easier for software agents than common-sense reasoning.

- The agents are less capable of picking travel arrangements fitting customer requirements up front. Instead they explore and generate many more options. Moreover, they keep on searching during execution and modify reservations when opportune (e.g., to handle a disturbance).

\subsubsection{Exploration and intention propagation by ant agents}

Based on the above scheme and inspired by food foraging in ant colonies, the holonic MES introduces two types of lightweight mobile agents, called ant agents. These ant agents, respectively, check resource availability and make/reconfirm reservations at the resources on behalf of the order agent that creates them.

The PROSA resource agents provide a navigation graph and the reservations infrastructure (Fig. 1). Each resource agent has a finite number of entries and exits. Entries are connected to exits of neighboring resources and vice versa. Exits and entries may also remain unconnected. Order agents are located in this resource graph. In the simplest case, this is the position of the not-yetfinished product instance, for which the order agent is responsible. From this position, the order agent creates ant agents, of both types, at a regular frequency.

First, the order agent creates exploring ant agents (Fig. 1). These ant agents virtually execute the remaining production steps, including all required handling and storage. During this exploration, resource agents receive inquiries about their availabilities and capabilities, which they answer based on the self-knowledge and their local reservations roster. No reservations or commitments are made. The ant agents stochastically explore different possibilities (each ant agent investigates exactly one possible solution). The mechanisms deciding which options will be explored are considered to be a variable part of the holonic MES design, which is continuously adapted and updated to account for the prevailing conditions. Their details are irrelevant in this paper. Stronger, if these details would be required for this discussion, the applicability of the technology in this paper would shrink and its scientific contribution would become anecdotal.

Second, the order agent creates intention ant agents (Fig. 1). This activity starts when an adequate number of possible solutions have been reported back by the exploring ant agents (details are irrelevant in the discussion). From these candidate solutions, the order agent picks its preferred solution: its intention. The intention ant agents virtually execute this solution and make/reconfirm the corresponding reservations at the resource agents involved. These reservations permit the resource agent to self-forecast the future load, which in turn allows the order agents to predict their, routing including timing.

The above procedure is executed continuously. When disturbances occur, the ant agents discover this, and order agents may adapt their intentions accordingly. Measures to manage the system dynamics are indicated but outside the scope of this paper $[18,19]$. The next section discusses how this basic and reusable schema is elaborated on to prefer executing an externally provided schedule over other alternatives reported by exploring ant agents. 


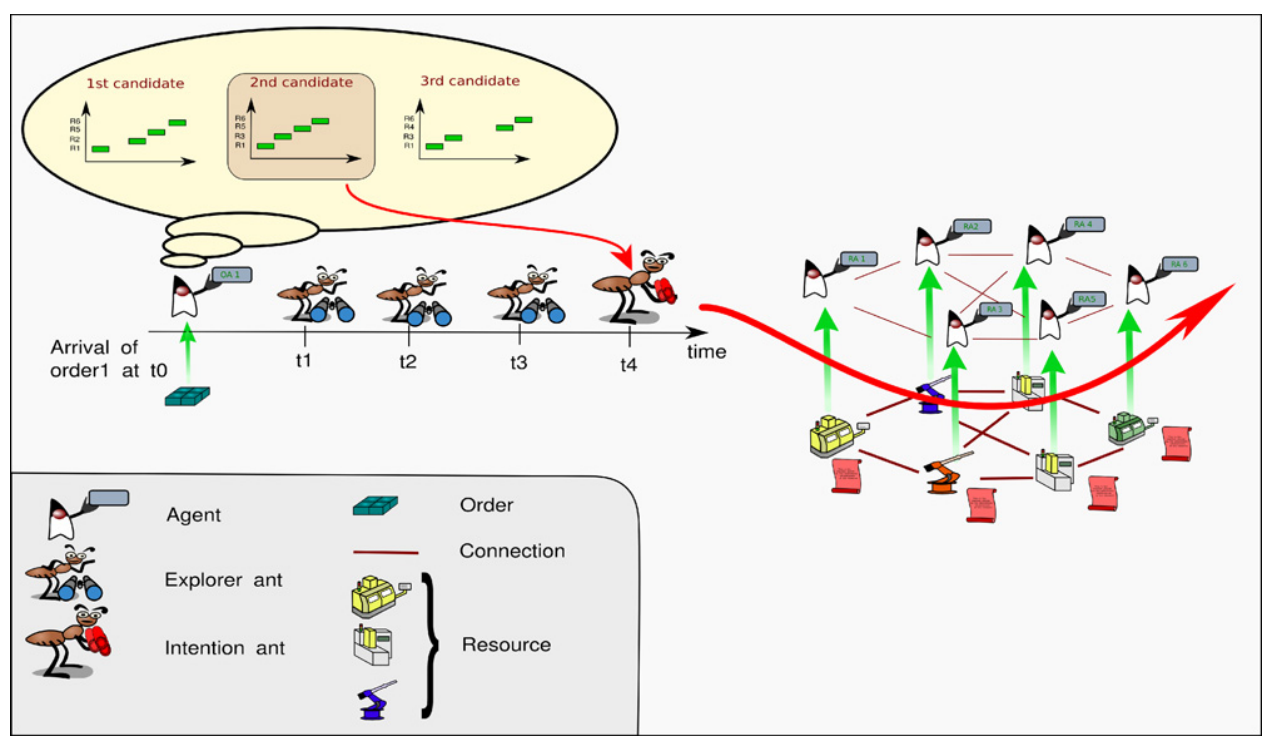

Fig. 1. Exploration and intention propagation by ant agents.

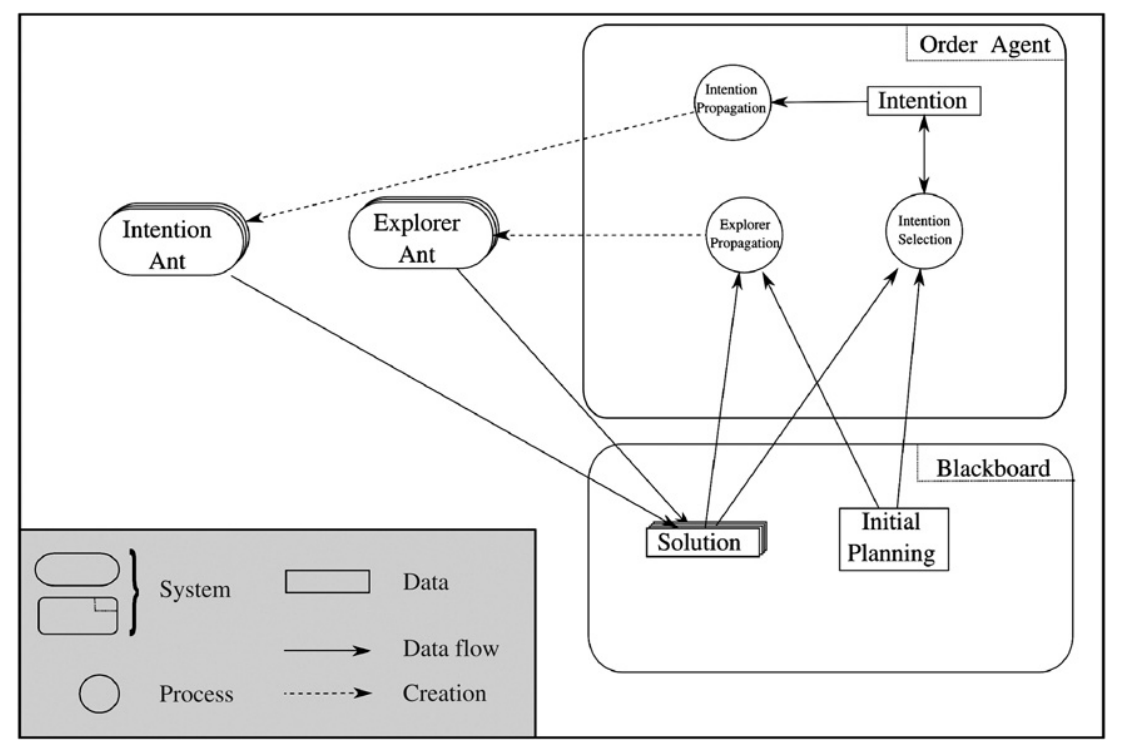

Fig. 2. Order agent run-time view.

\subsection{Autonomic schedule execution}

In this first development, the selection of intentions by the order agents is biased toward executing a given schedule. The exploration for possible solutions remains unbiased, guaranteeing that feasible solutions will be found even if the schedule is infeasible (that is, autonomic manufacturing control). In future developments, a given percentage of the exploring ant agents will be biased toward routings and timings in the given schedule, and resource agents will grant more priorities to requests in accordance with the given schedule.

The modification to the basic holonic MES - to achieve autonomic schedule execution - affects the selection of the initial intention by an order agent as well as the eventual changing of this intention. Fig. 2 shows a run-time view of the modified order agent. To implement the decision-making mechanism biased toward the given schedule, the order agent executes the following steps:

1. The order agent retrieves solutions discovered and sent back by its exploring ant agent.
2. The order agent ranks these solutions and its current intention in accordance with its performance criterion biased toward the given schedule (explained below). The order agent marks the top-ranking solutions as the candidate intention.

3. If the current intention of the order agent already exists and has been successfully refreshed (that is, no disturbance has rendered the solution invalid), the order agent switches its intention to the candidate intention with a $60 \%$ probability. Otherwise, the current intention is kept. If the order agent has no valid current intention, the candidate intention becomes the current intention with a $90 \%$ probability.

4. If the order agent decides to change intentions, it first checks whether the candidate intention still is a valid, top-ranking solution by means of an exploration refresh (not reserving resource slots).

5. The current intention (changed or not) is propagated by means of creating and sending out an intention ant agent.

To bias the manufacturing execution toward the given schedule, the ranking in step 2 utilizes a criterion that combines deviation from this schedule with application-oriented performance 
measurements (e.g., lead time, tardiness, bottleneck utilization). In particular, the current research prototype uses:

$R(s)=\max \left(100-\sum_{t=0}^{n}\left(e_{t}^{s}-e_{t}^{p}\right) / d_{t}^{p} \times 100,0\right)$

when the solution is using the machines specified by the planning; otherwise,

$R(s)=0$

where $t$ is a task in solution $s$ (out of $n$ tasks)

$e_{t}^{s}$ is the end of the task in the solution

$e_{t}^{p}$ is the end of the task in the planning

$d_{t}^{p}$ is the duration of the task in the planning.

This measure prefers solutions that are executed on the machines indicated by the schedule, and solutions matching finishing times approaching the schedule times. In case of multiple solutions having the same value for $R(s)$, the solution with the shortest lead time is chosen. Note that in case of large deviations, $R(s)$ will be zero, and lead time decides the selection of the current intention.

Note that there is no research contribution in the specific details of the above. It is a very basic mechanism that makes the MES follow the advice (schedule) on the condition that it is feasible within certain boundaries. Optimization of such boundaries is outside the scope of our research. Moreover, the gigantic size of the design space for the MES and its decision mechanisms implies that a concrete industrial case will be required to reduce this space to a more manageable size. Note that every order in this system may apply a customized version of the decision rule and modify its rule during execution. In industrial practice, it is common to have multiple order types-urgent, normal, and so on. Also, a disturbance may cause rework and thus may change the affected order from normal into urgent. The search/design space definitely is challenging.

\section{Experimental results}

This section describes how the modifications to achieve autonomic schedule execution affect the holonic MES behavior. In particular, the experiments examine which schedules are followed by the MES and which are not followed for a given case. Therefore, the experiments vary the schedules given as input to the MES, keeping all other parameters constant. Section 4.1 describes this case, which is a simplified version of a real manufacturing case. Section 4.2 describes the input and output parameters chosen. The final section presents and discusses the results.

\subsection{Case study}

This section describes the production system [20]. This involves the structural features (e.g., the presence of equipment) and the technological features (e.g., processes supported by this equipment). The production system is a scaled-down version of an existing factory (confidentiality prevents the use of an exact representation). Regarding structural features, the production plant is a job shop, containing workstations, intended to produce the long parts of weaving machines.

As shown in Fig. 3, the plant comprises four workstations, a transporting unit, and a warehouse. The transporting unit moves containers, each containing an order, throughout the production system. All workstations and warehouse slots are connected to the transporting unit. In addition, the transporting unit is connected to the entrance and the exit of the plant. It is capable of transporting a container from and to all of its connected units, that is, the warehouse, the workstations, the entrance, and the exit.
Table 4

Scheduled performance: lead time

\begin{tabular}{llll}
\hline Slack - Number of orders & 0 & 50 & 100 \\
\hline 3 & 350 & 450 & 550 \\
5 & 350 & 450 & 550 \\
7 & 350 & 450 & 550 \\
\hline
\end{tabular}

The warehouse consists of several racks. Each rack consists of several storing places (storage slots). The warehouse can hold 90 containers in total. Three out of four workstations in the plant are milling machines. Two milling machines, W3664 and W3665, are capable of fine milling. The third milling machine, W3824, is only capable of rough milling. The fourth and last machine, W9038, is a cleaning station: the long parts are cleaned there after they have been milled.

The technological features definition provides the process plans that can be performed in the job shop. The process plan in this scenario consists of three steps: rough milling, fine milling, and cleaning. Some operations can be performed on more than one machine, but their sequence cannot be altered. In this scenario, all orders require the same processing time on a machine. The rough milling operation on W3824 takes $100 \mathrm{~min}$, the fine milling operation on W3664 and W3665 takes $200 \mathrm{~min}$, and the cleaning operation takes $50 \mathrm{~min}$.

\subsection{Experimental design}

This experiment examines the performance of the holonic MES, with different given schedules. This research refrains from developing or studying the scheduling systems themselves. The schedules are a given input to the system.

The schedule specifies which job need to be performed on which machine in what time period. They do not specify details such as which transportation unit to use for carrying parts from and to the machine, where to store parts, which tools to use to manufacture parts, and so on. These details are highly dependent on the manufacturing dynamics and are therefore unknown up front.

The schedules vary according to two parameters, which are the slack in the schedule and the number of orders in the schedule. Given these two parameters, a schedule with minimal lead time is created. Because the schedule does not specify transportation or storage of parts, creating the optimal schedule remains simple and has been performed manually.

A factorial design [21] is used in this experiment. A period of one week (or five shifts or $2450 \mathrm{~min}$ ) is studied. To study the effect of possible transportation delays, the run length is extended to $3000 \mathrm{~min}$.

For one week, the maximum number of orders was set to seven, that is, the studied period divided by the minimal lead time ( 350 min). The minimum number of orders was chosen to be three. An intermediate setting - five orders - completes the experimental setup. The maximum slack was chosen to be half of the maximum processing time, that is, 100 min. The minimum was chosen to be $0 \mathrm{~min}$ and the center value to be 50 .

Output parameters considered were lead time and utilization. Lead time is the performance criterion, optimized by the given schedules. Utilization is important to machine operators (e.g., depending on the machine used, different operators may be needed). Table 4 gives the scheduled lead-time values for the various input parameters. Table 5 does the same for utilization of W3664.

\subsection{Results}

For each combination of the input parameters (as indicated in Table 4), 15 replications were performed ( 135 replications in total). The results are summarized in Figs. 4 and 5. 


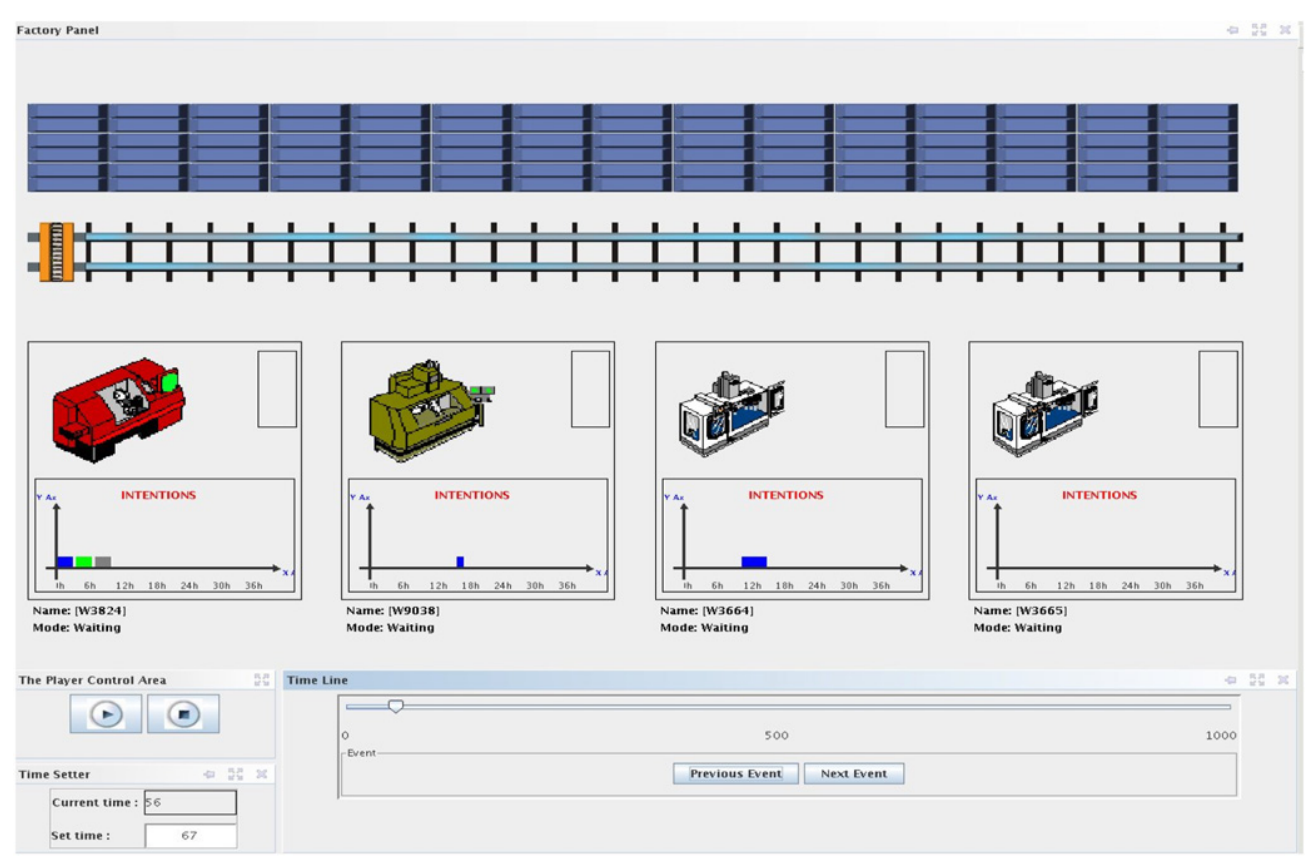

Fig. 3. Plant layout for the experiments.

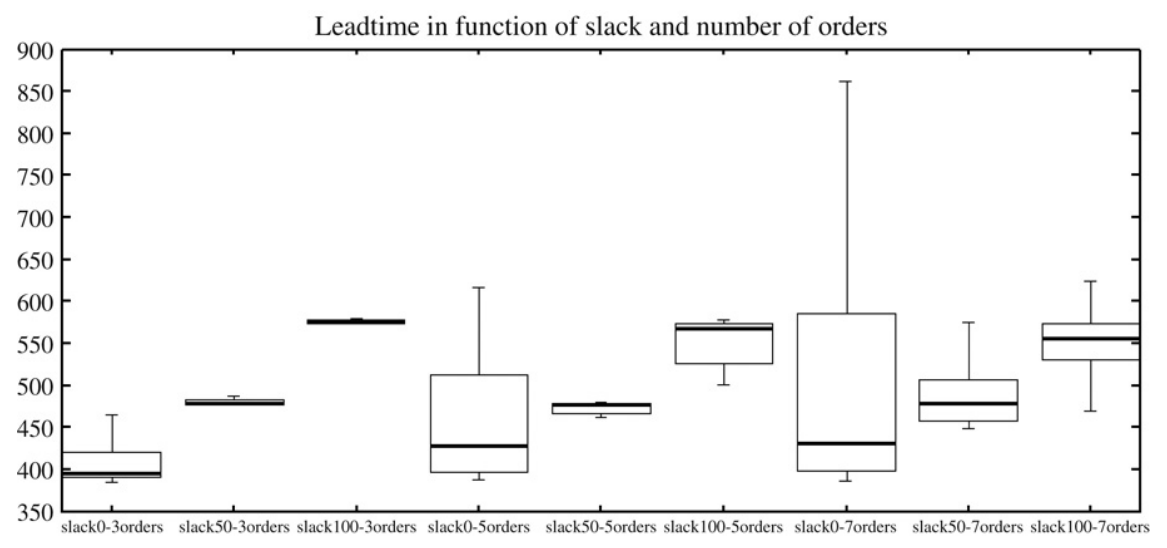

Fig. 4. Lead time for schedule execution.

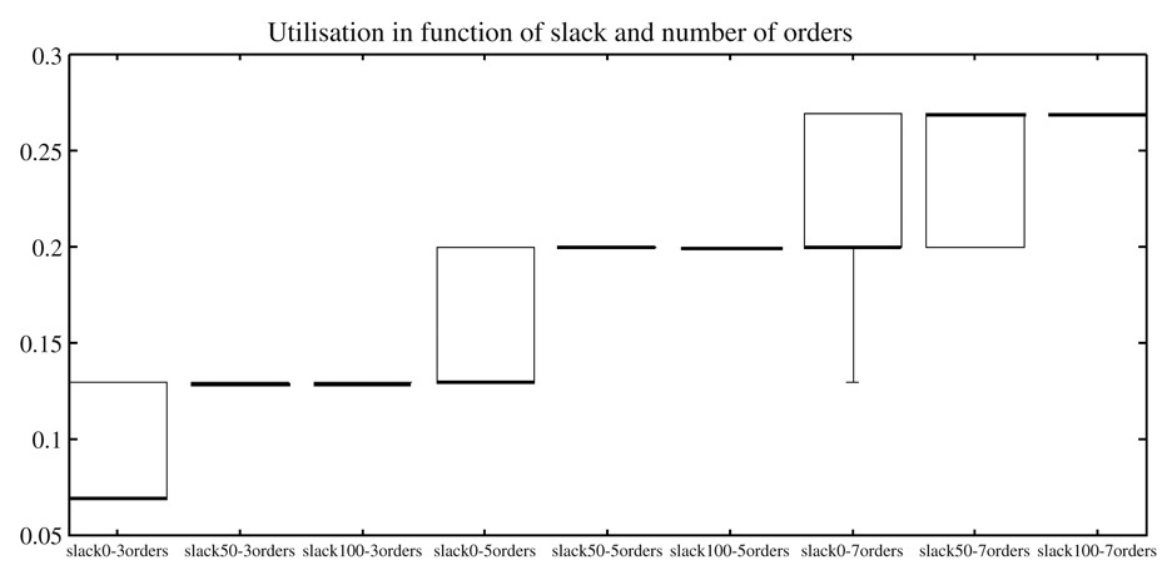

Fig. 5. W3664 utilization for schedule execution.

\subsubsection{Lead time}

Fig. 4 shows the evolution of lead time as a function of the number of orders and slack in the given schedule. For each combination of parameters, a box plot is displayed. The black line represents the median.
For slack 0 , the median is higher than the scheduled value (350) and the variation is large. These schedules are not followed in most cases. The schedule neglects to account for transportation, which makes it impossible to respect the given start and finishing times. The orders have to self-organize to optimize lead time. This means 
Table 5

Scheduled performance: utilization of W3664

\begin{tabular}{llll}
\hline Slack - Number of orders & 0 & 50 & 100 \\
\hline 3 & 0.13 & 0.13 & 0.13 \\
5 & 0.2 & 0.2 & 0.2 \\
7 & 0.27 & 0.27 & 0.27 \\
\hline
\end{tabular}

that some orders neglect the planning and use the performance parameter provided as described in Section 3.

Increasing slack decreases the difference between planned and actual lead time. For slack 50, this gives an average difference of $32.6 \mathrm{~min}$, with a standard deviation of $25.9 \mathrm{~min}$. For slack 100, the average difference is $11 \mathrm{~min}$, with a standard deviation of $28.9 \mathrm{~min}$. This is smaller than the average difference for slack 0 , which is 127 min with a standard deviation of $146 \mathrm{~min}$.

The lead time is not constant, even when increasing the slack. This means that some orders still deviate from the planning. This can be explained by the fact that increasing slack does not decrease the peak demand on the transportation unit. Indeed, all orders are shifted over an equal time period. For instance, order 1 is scheduled to finish its operation at W3824 at time 100. After that, two operations need to be performed at the same time, namely starting order 1 at the next machine, W3664, and starting the next order, order 2, at W3824. Introducing slack will shift both operations by an equal amount. The transportation unit still needs to handle both orders at the same time.

For a high number of orders and high slack, the median in lead time is lower than the planned value. Orders can easily find better solutions when the planned lead time is large. The more orders, the higher the chance that they will search a solution themselves instead of following the planning, because of demand peaks on the transportation unit.

Increasing the number of orders increases the variation in lead time. When orders need to search for a solution themselves, the number of solutions provided to them is dependent on the number of exploring ants sent. This number of exploring ants remains constant, while the search space increases when increasing the number of orders. Therefore, it becomes harder for the order agents to find good solutions.

\subsubsection{Utilization}

Fig. 5 shows the evolution of the utilization as a function of the number of orders and slack for W3664. The paper shows the utilization for this machine because there is an alternative, W3665. For W3824 and W9038, there are no alternatives, so utilization remains the same if all orders are completed in time. For slack 50 and slack 100 , all planned values (see Table 5 ) are always obtained, except for slack 50 with seven orders. For slack 0, planned values are not always obtained.

The fact that there is variation in lead time for slack 50 and slack 100 (where the planning is feasible), while there is almost no variation in utilization, shows that changing lead time is preferred over changing machines. However, if the number of orders increases, the amount of slack needed to handle delays increases. For slack 50 and seven orders, the slack time does not suffice anymore, to avoid changing from the scheduled machine to its alternative.

As mentioned in the previous section, schedules with slack 0 are unfeasible. Therefore, almost all orders will search for a new solution. Some orders might take an earlier slot. The deviation (shift in time) for other orders then becomes so big that it becomes equal to using another machine.

\subsubsection{Discussion}

The experiments show that the MES follows feasible schedules. In contrast, the MES is not (yet) able to approximate unfeasible schedules well. Moreover, when the MES search space grows (more orders, more machines), the current MES is likely to experience increasingly more difficulties when it occasionally fails to discover suitable routings that follow the given schedule. The latter will be remedied by having exploring ant agents prefer discovering routings that stick to the schedule (future work).

The experiments indicate that inserting a fixed amount of slack has drawbacks:

- The best choice for the amount of slack depends on the performance criterion. Minimizing lead-time deviation calls for slack 50 whereas minimal deviation for utilization indicates slack 100.

- The best choice for the amount of slack depends on the load of the system (number of orders).

- The best choice depends on the ability of the MES system to discover suitable routings.

Overall, the amount of slack would have to be tuned regularly. Note that a more intelligent solution would provide more insight on the purpose of the information in the given schedule, allowing the execution system to assess which deviations are important (to be avoided) and which are not [11]. Such an approach is more likely to solve the problem than never-ending tuning of the slack parameter.

This intelligent solution brings up the question of what it means to follow a schedule and how to do this. First of all, there is the trade-off between time and place. Should the MES prefer sticking to the timing or to the machine assignment in the schedule? Should the MES minimize a weighted overall deviation from schedule, or should it prefer rejoining the schedule at some future point (by deviating completely from schedule in the immediate future)? What does it mean to rejoin the schedule?

Overall, these initial results show that a schedule can guide the MES, but that robust and scalable solutions call for further developments. An important issue is to capture and/or encapsulate what it means to deviate from a given schedule. Subsequently, the MES can provide suitable answers to the schedule deficiencies.

\section{Conclusions and future work}

This paper presents a holonic MES that uses a given schedule as a guideline for selecting among task execution alternatives. It is an autonomic design, which means that the MES generates solutions that are independent from the given schedule. This ensures that the MES is able to find solutions even when the schedule is infeasible. And, it generates alternative solutions allowing assessment of schedule quality.

The experiments show that the MES follows feasible schedules but is not (yet) able to approximate infeasible schedules. The research reveals the need to encapsulate what it means to 'stick to the schedule' such that the MES can make informed choices when it cannot follow the schedule exactly. Also, scalability needs more guarantees that solutions along the schedule will be discovered and evaluated by the MES.

Here, future research includes:

- The design and implementation of order agents that equip a significant percentage (e.g., 30\%) of their exploring ant agents with a selection mechanism that is biased toward the given schedule. This ensures that deviations from the given schedule are unlikely to be caused by the failure to discover suitable solutions. 
- Encapsulating the criterion indicating schedule compliance. This enables the schedule provider to decide what the distance to the given schedule is, and allows situation-dependent interpretation of, for instance, the available slack in the schedule.

- Investigating different schedule-compliance criteria, including criteria that emphasize 'rejoining of the schedule' over 'trying to stick to the schedule' in case of disturbances.

- Systematic investigation of schedule parameters such as its quality, feasibility, and robustness.

- Capturing current practice in a pheromone trail as an alternative input for the given schedule. In industry, using current practice as a reference solution is quite common.

- An industrial case study, in which the above pheromone trail will be used.

- Enhancing the autonomic capabilities of the order agents, allowing them to interpret the quality and validity of the given schedule more intelligently.

Overall, this paper discusses a first development in having a decentralized manufacturing control system utilize an externally provided schedule while maintaining the robustness and completeness of the MES. The design of the MES, postponing the schedule execution decisions until the final stages of the implementation, has been quite helpful: it allows implementing experiments without having to adapt the MES software except for the decision mechanisms themselves. Finally, many interesting research issues and development challenges remain, including the question to what extent schedulers and the MES system can be made cooperative while avoiding their respective weaknesses and safeguarding their respective assets.

\section{Acknowledgments}

This paper presents work funded by the Research Fund of the K.U. Leuven (Concerted Research Action on Autonomic Computing for Distributed Production Systems).

\section{References}

[1] Duffie N. Synthesis of heterarchical manufacturing systems. Computers in Industry 1990;14:167-74.

[2] Bussmann S, Schild K, Self-organizing manufacturing control: An industrial application of agent technology. In: Proc. 4th int. conf. on multi-agent systems. 2000. p. 87-94.

[3] Lin GY, Solberg JJ. Integrated shop floor control using autonomous agents. IIE Transactions 1992;24(3):57-71.

[4] Parunak HVD, Baker AD, Clark SJ, The AARIA agent architecture: An example of requirements-driven agent-based system design. In: Proc. 1st int. conf autonomous agents. 1997. p. 482-3.

[5] Márkus A, Kis T, Váncza J, Monostori L. A market approach to holonic manufacturing. Annals of the CIRP 1996;45(1):433-6.

[6] Monostori L, Váncza J, Kumara S. Agent-based systems for manufacturing. Annals of the CIRP - Manufacturing Technology 2006;55(2):1-24

[7] Valckenaers P, Van Brussel H. Holonic manufacturing execution systems. CIRP Annals-Manufacturing Technology 2005;54(1):427-32.

[8] Csáji BCs, Monostori L, Kádár B, Learning and cooperation in a distributed market-based production control system. In: Proc. of 5th int. workshop on emergent synthesis. 2004. p. 109-17.

[9] Valckenaers P, Van Brussel H, Hadeli, Bochmann O, Saint Germain B, Zam rescu $C$. On the design of emergent systems: An investigation of integration and interoperability issues. Engineering Applications of Artificial Intelligence 2003;6(4):377-93.

[10] Smith RG. The contract net protocol: High-level communication and control in a distributed problem solver. IEEE Transactions on Computers 1988;C-29(12): 357-66.

[11] Bongaerts L, Monostori L, McFarlane D, Kádár B. Hierarchy in distributed shop floor control. Computers in Industry 2000;43(2):123-37.

[12] Leitao P, Colombo AW, Restivo FJ. ADACOR: A collaborative production automation and control architecture. IEEE Intelligent Systems 2005;20(1): 58-66.

[13] Ueda K, Lengyel A, Hatono I. Emergent synthesis approaches to control and planning in make to order manufacturing environments. Annals of the CIRP 2004;53(1):385-8
[14] Parunak HVD, Weyns D. Guest editors' introduction, special issue on environments for multi-agent systems. Autonomous Agents and Multi-Agent Systems 2007;14(1):1-4

[15] Weyns D, Omicini A, Odell J. Environment as a first class abstraction in multiagent systems. Autonomous Agents and Multi-Agent Systems 2007 14(1):5-30.

[16] Valckenaers P, Hadeli, Saint Germain B, Verstraete P, Van Belle J, Van Brussel H. From intelligent agents to intelligent beings. In: Mařík V, Vyatkin V, Colombo AW, editors. HoloMAS 2007. LNAI, vol. 4659. Berlin Heidelberg: Springer-Verlag; 2007. p. 17-26.

[17] Valckenaers P, Hadeli, Saint Germain B, Verstraete P, Van Brussel H. Emergen short-term forecasting through ant colony engineering in coordination and control systems. Advanced Engineering Informatics 2006;20:261-78.

[18] Hadeli, Valckenaers P, Verstraete P, Saint Germain B, Van Brussel H. A study of system nervousness in multi-agent manufacturing control system. Engineering Self-Organizing Systems 2005;232-43.

[19] Hadeli, Bio-inspired multi-agent manufacturing control systems with socia behavior. Ph.D. K.U. Leuven, July 2006.

[20] Cavalieri S, Macchi M, Valckenaers P. Benchmarking the performance of manufacturing control systems: Design principles for a web-based simulated test-bed. Journal of Intelligent Manufacturing 2003;14(1):43-58.

[21] Montgomery D. Design and analysis of experiments. New York: John Wiley \& Sons; 1998.

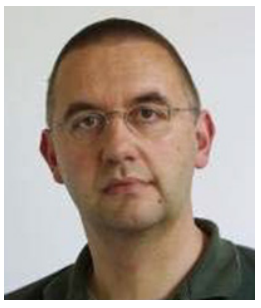

Paul Valckenaers received the applied mathematics engineering, computer science engineering, and mechanica engineering $\mathrm{Ph}$. D degree from the K.U. Leuven, Belgium. He is an Industrial Research Fellow in the mechanical engineering department of the K.U. Leuven.

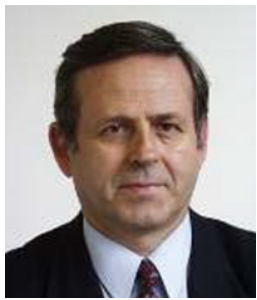

Hendrik Van Brussel is full professor at the Faculty of Engineering of the K.U. Leuven. He received his M.Sc.EE and Ph.D. degrees from K.U. Leuven, Belgium. He is Fellow of SME and IEEE and he received honorary doctor degrees from the 'Politehnica' in Bucarest and from RWTH Aachen. $\mathrm{He}$ is a member of the Royal Flemish Academy of Belgium for Sciences and Fine Arts, past president of the CIRP. and foreign member of the Royal Swedish Academy of Engineering Sciences.

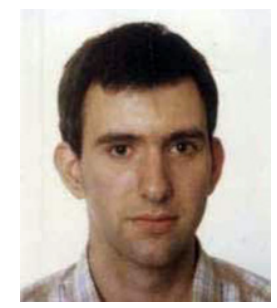

Paul Verstraete received the software engineering degree from the K.U. Leuven, Belgium. He also obtained a degree in industrial economics from the same university. He is a candidate for the Ph.D. degree in mechanical engineering from the K.U. Leuven. Since 2003, he has been with the Mechanical Engineering Department, division PMA, of the K.U. Leuven.

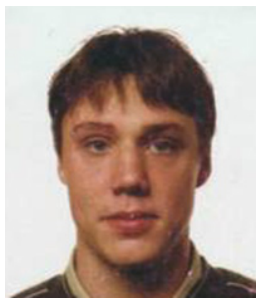

Bart Saint Germain received the software engineering degree from the K.U. Leuven, Belgium. He is a candidate for the Ph.D. degree in mechanical engineering from the K.U. Leuven. Since 2002, he has been with the Mechanical Engineering Department, division PMA, of the K.U. Leuven.

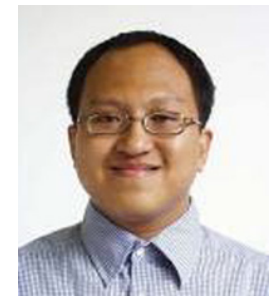

Hadeli received the industrial engineering degree from the Parahyangan Catholic University, Indonesia. $\mathrm{He}$ obtained the master's degree in industrial engineering and engineering management from the Institute Technology of Bandung, Indonesia, and master's degree and Ph.D. in engineering from the K.U. Leuven. Belgium. Since 2000, he has been with the Mechanical Engineering Department, division PMA, of the K.U. Leuven. 